\title{
Quantum nonlocal effects in individual and interacting graphene nanoribbons
}

\author{
Iván Silveiro $^{1}$, Juan Manuel Plaza Ortega ${ }^{1}$ and F Javier García de Abajo ${ }^{1,2}$
}

We show that highly doped graphene ribbons can support surface plasmons at near-infrared frequencies when their width is in the nanometer range, leading to important nonlocal and finite quantum-size corrections, such as sizable blueshifts. The magnitude of these effects is assessed by comparing classical and quantum-mechanical models to describe graphene plasmons. More precisely, we examine individual and interacting 6-8 nm wide zigzag and armchair ribbons doped to 0.4-1.5 eV Fermi energies. We find a strong influence of nonlocal effects on the orientation of graphene edges, with plasmons in zigzag ribbons undergoing strong quenching when their energy is below the Fermi level. Nonlocality is also affecting the hybridization between ribbon plasmons in dimers and arrays for separations below a few nanometers. Remarkably, the removal of a single row of atomic bonds in a ribbon produces a strong plasmon frequency shift, whereas the removal of bonds along an array of rows separated by several nanometers in an extended sheet causes a dramatic increase in the absorption. Besides the fundamental interest of these results, our work supports the use of narrow ribbons to achieve electro-optical modulation in the near infrared.

Light: Science \& Applications (2015) 4, e241; doi:10.1038/lsa.2015.14; published online 30 January 2015

Keywords: graphene; graphene plasmons; graphene ribbons; metamaterials; plasmonics

\section{INTRODUCTION}

Collective oscillations of conduction electrons, also known as plasmons, find a vast number of applications in nanophotonics due to their ability to enhance and confine optical fields far below the wavelength scale. ${ }^{1}$ In particular, plasmons supported by noble metal nanostructures have been extensively used for optical trapping, ${ }^{2}$ nonlinear optics, ${ }^{3-5}$ single-molecule detection ${ }^{6,7}$ and signal processing, ${ }^{8}$ among other feats. Recently, highly doped graphene has also been found to sustain plasmons at infrared and lower frequencies, ${ }^{9-19}$ with the unique advantages of possessing a large electrical tunability, long lifetimes, and strong nonlinearities. ${ }^{20-23}$ The peculiar electronic structure of this two-dimensional material ${ }^{24,25}$ allows us to substantially shift its Fermi level ( $E_{\mathrm{F}}=\hbar v_{\mathrm{F}} \sqrt{\pi n}$, where $v_{\mathrm{F}} \approx 10^{6} \mathrm{~m} \mathrm{~s}^{-1}$ is the Fermi velocity) by adding a moderate density of doping charge carriers $n$. Fermi energies as high as $\sim 1 \mathrm{eV}$ have been achieved through electrostatic gating. ${ }^{26,27}$ Furthermore, strong coupling of light to localized plasmons can be realized by patterning the graphene, ${ }^{28,29}$ even reaching the limit of complete optical absorption. ${ }^{30}$ While a classical electromagnetic description of plasmons is sufficient to deal with large graphene structures compared with the Fermi wavelength $\sqrt{4 \pi / n}, 9,16,28,31-35$ nonlocal quantum effects play an important role in smaller islands, ${ }^{36}$ including plasmon quenching through coupling to electronic zigzag edge states, ${ }^{37,38}$ which is reminiscence of the quenching described in near-touching metallic nanoparticles. ${ }^{39,40}$

In this work, we study nonlocal quantum effects in individual and interacting graphene structures. We concentrate on nanoribbons as they are central elements in many previous theoretical ${ }^{28,34,37,38,41-43}$ and experimental ${ }^{10,13,14,18,43}$ graphene plasmon studies, and also stimulated by the availability of new methods of synthesis with control over the ribbon width down to the nanometer scale. ${ }^{4-47}$ Additionally, ribbons can be electrically contacted far from the region in which plasmons are exploited, thus enabling optical tunability using non-invasive electrical gating. In particular, we compare classical and quantum-mechanical models for the graphene optical response, focusing on the lowest-order dipole plasmons and examining their role in the normal-incidence extinction cross-section for light polarized perpendicular to the ribbon edges. We study ribbons of $<10 \mathrm{~nm}$ width and different edge terminations - armchair (AC) and zigzag (ZZ) — as well as their interactions in closely spaced dimers and periodic arrays. Our results provide a roadmap of plasmons in individual and interacting narrow ribbons, reaching near-infrared energies and exhibiting important nonlocal effects that must be considered in the design of potential device applications. These results also reveal the advantage of ribbons relative to other alternative methods to exploit near-infrared plasmons, such as gratings (they should have similar periodicity as the ribbon widths here considered), tips and local emitters (they generally couple non-resonantly to propagating plasmon bands, thus resulting in broad spectral features), and prisms (the large mismatch between light and graphene-plasmon wavelengths requires the use of very high-index dielectrics that are currently unavailable in the near infrared).

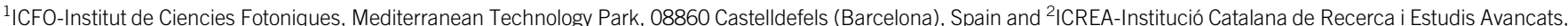
Passeig Lluís Companys 23, 08010 Barcelona, Spain

Correspondence: Professor FJ García de Abajo, ICFO-Institut de Ciencies Fotoniques, Mediterranean Technology Park, 08860 Castelldefels (Barcelona), Spain

E-mail: javier.garciadeabajo@icfo.es

Received 13 August 2014; revised 11 November 2014; accepted 13 November 2014; accepted article preview online 19 November 2014 


\section{MATERIALS AND METHODS}

The extinction cross-section, which we present normalized to the graphene area, is obtained through the optical theorem from the ribbon polarizability, which we in turn calculate from the induced density using the classical ${ }^{28}$ and quantum-mechanical ${ }^{38}$ methods described elsewhere. In particular, the quantum description, which we term TB-RPA model, is based upon the random-phase approximation ${ }^{48}$ (RPA) using tight-binding (TB) electronic states as input. ${ }^{38}$ However, in the classical electromagnetic model the carbon layer is represented by a local conductivity, which is taken from the local-RPA approximation $^{28}$ and used as input to solve the Maxwell equations with the boundary element method. ${ }^{49}$

In the quantum-mechanical calculations presented below for ribbon arrays, the absorbance is obtained from the effective polarizability per unit length $\tilde{\alpha} / L$ for each ribbon, which we in turn obtain from the quantum TB-RPA model, conveniently modified to deal with infinite ribbon arrays (see below). This model yields the non-interacting susceptibility $\chi_{l l^{\prime}}^{0}$, which permits writing the induced density as $\rho_{l}=\sum_{l^{\prime}} \chi_{l l^{\prime}}^{0} \phi_{l^{\prime}}$ in terms of the total potential $\phi_{l}$, where $l$ and $l$ ' run over the carbon sites of the graphene structure. ${ }^{41}$ The total potential is related to the external potential $\phi_{l}^{\text {ext }}$ through $\phi_{l}=\phi_{l}^{\text {ext }}+\sum_{l^{\prime}} v_{l l^{\prime}} \rho_{l^{\prime}}$, where $v_{l l}$ is the Coulomb interaction between sites $l$ and $l$ '. These expressions can be easily combined to find the induced density as $\rho=\left(1-\chi^{0} \cdot v\right)^{-1} \cdot \chi^{0} \cdot \phi^{\text {ext }}$, using matrix notation. For a finite number of ribbons, the one-dimensional periodicity of the atomic lattice is used to reduce the calculation to a finite number of sites ( $l$ and $l$ ' indices) within a unit cell. ${ }^{41}$ Here, for ribbon arrays, we follow this procedure to calculate $\chi^{0}$ for a single ribbon, but supplementing $v_{l l}$ with the sum of all interactions of site $l$ with the equivalent $l$ ' sites in all other ribbons. The effective polarizability per unit length is then given by $\tilde{\alpha} / L=\sum_{l} x_{l} \rho_{l} /\left(E^{\text {ext }} b\right)$, where the sum extends over the noted unit cell of length $b$ along the ribbon, $x_{l}$ is the distance across it, and $E^{\text {ext }}$ is the external electric field (i.e., $\phi_{l}^{\text {ext }}=-x_{l} E^{\text {ext }}$ ). Finally, the normalincidence reflection and transmission coefficients reduce to $r=(2 \pi \mathrm{i} k / a)(\tilde{\alpha} / L)$ and $t=1+r$, where $a$ is the period of the array, and from here, the absorption reduces to $1-|r|^{2}-|1+r|^{2} .^{35}$

For simplicity, we assume that the ribbons are in vacuum at $300 \mathrm{~K}$ in both the classical and quantum calculations. The addition of a substrate of permittivity $\epsilon_{\mathrm{s}}$ can be simply introduced through an effective permittivity $\epsilon_{\text {eff }}=\left(\epsilon_{\mathrm{s}}+1\right) / 2$ of the surrounding medium. This is a rigorous correction in the local electrostatic limit, ${ }^{41}$ which essentially leads to a redshift of plasmon frequencies by a factor $\approx 1 / \sqrt{\epsilon_{\mathrm{eff}}}$.

\section{RESULTS AND DISCUSSION}

\section{Individual ribbons}

We start by analyzing plasmons in individual ribbons of small width $W=6 \mathrm{~nm}$ (Figure 1). The transverse dipole plasmon on which we concentrate here is the lowest-energy feature in the spectra. Remarkably, the classical calculations (insensitive to the edge configuration) are in excellent quantitative agreement with quantum-mechanical calculations for both $\mathrm{AC}$ and $\mathrm{ZZ}$ edges provided the plasmon energy is below the Fermi level (see $E_{\mathrm{F}}=1 \mathrm{eV}$ and $1.5 \mathrm{eV}$ curves in Figure 1). If this condition is not satisfied $\left(E_{\mathrm{F}}=0.4 \mathrm{eV}\right)$, the plasmon is strongly quenched in the ZZ-edge ribbons, while it is slightly shifted and broadened in AC-edge quantum calculations in comparison with classical theory. This behavior is fully reproducible over a large range of Fermi energies and small ribbon widths, of which Figure 1 shows some representative examples. The observed plasmon quenching can be attributed to the existence of zero-energy electronic edge states, as already shown for even narrower ribbons. ${ }^{37}$ Additionally, the plasmon

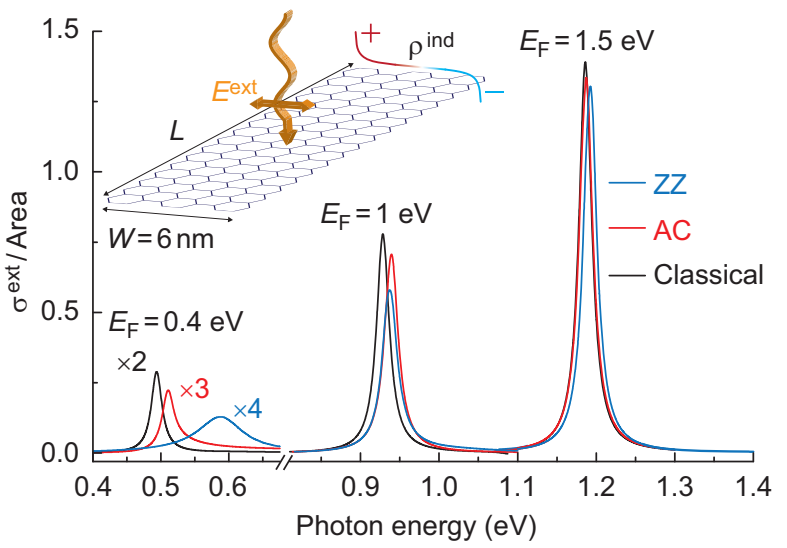

Figure 1 Plasmons in individual graphene ribbons. We show the normal-incidence extinction cross-section of self-standing graphene nanoribbons (width $W=6 \mathrm{~nm}$ and intrinsic damping of $0.02 \mathrm{eV}$ ) for several values of the Fermi energy $E_{\mathrm{F}}$, calculated using two different models: classical theory with the local-RPA conductivity; ${ }^{28}$ and quantum-mechanical TB-RPA for ribbons with $\mathrm{ZZ}$ and $\mathrm{AC}$ edges. ${ }^{38}$ We focus on the lowest-order dipolar mode (see induced charge density in inset), which is excited by light polarized across the ribbon. AC, armchair; RPA, random-phase approximation; TB, tight-binding; ZZ, zigzag.

energy increases with $E_{\mathrm{F}}$, following the approximate behavior ${ }^{35}$ $\hbar \omega_{\mathrm{p}} \approx 4 e \sqrt{E_{\mathrm{F}} / \pi W}$ (this expression is obtained from the Drude model, while RPA corrections push the plasmon energy slightly downwards), whereas its strength grows with doping as well.

\section{Ribbon dimers}

Two doped coplanar parallel ribbons separated by a carbon-to-carbon edge distance $d$ interact producing significant changes in the plasmonic spectrum of the whole structure. ${ }^{41}$ We study this configuration in Figure 2 for ribbons of width $W=6 \mathrm{~nm}$ and Fermi energy $E_{\mathrm{F}}=0.4 \mathrm{eV}$ using both classical (upper part) and quantum TB-RPA (lower part, for $\mathrm{AC}$ edges) theories. The interaction is attractive and leads to redshifts in the dipole-active bonding plasmons here discussed. ${ }^{41}$ The sequence of spectra shows that the discrepancy between classical and quantum calculations increases as the ribbons approach each other. This is consistent with the intuitive idea that nonlocal interactions gain weight at shorter distances. As part of this behavior, the quantum calculations do not seem to approach the plasmon of the double-width ribbon (left dashed-curve spectrum), in contrast to the classical simulations. The evolution of the dimer plasmon energy with separation (Figure 3) clearly illustrates this effect: there is a sizeable jump in the quantum calculation of the double-width AC plasmon when a centered row of C$\mathrm{C}$ bonds is removed (i.e., the TB hopping is set to zero in those bonds, represented as dashed lines in the left inset of Figure 3), thus becoming a closely spaced dimer. However, the classical calculation shows a continuous convergence of the dimer towards the double-width ribbon at small distances. Incidentally, as ribbon edges are generally passivated with hydrogens, and considering the $\mathrm{C}-\mathrm{H}$ and $\mathrm{H}-\mathrm{H}$ bond distances, we estimate that only carbon-to-carbon edge distances $>0.3 \mathrm{~nm}$ are physically realistic, with well-separated and non-tunneling electronic states in each ribbon. We include smaller distances for tutorial purposes, and in particular $d_{\min }=\sqrt{3} a_{0} / 2$ in AC ribbons in order to study the effect of removing one row of $\mathrm{C}-\mathrm{C}$ bonds, as discussed above.

\section{Periodic ribbon arrays}

A monolayer of extended undoped graphene exhibits a nearly constant absorption $\pi \alpha \approx 2.3 \%,{ }^{50,51}$ where $\alpha \approx 1 / 137$ is the fine-structure 

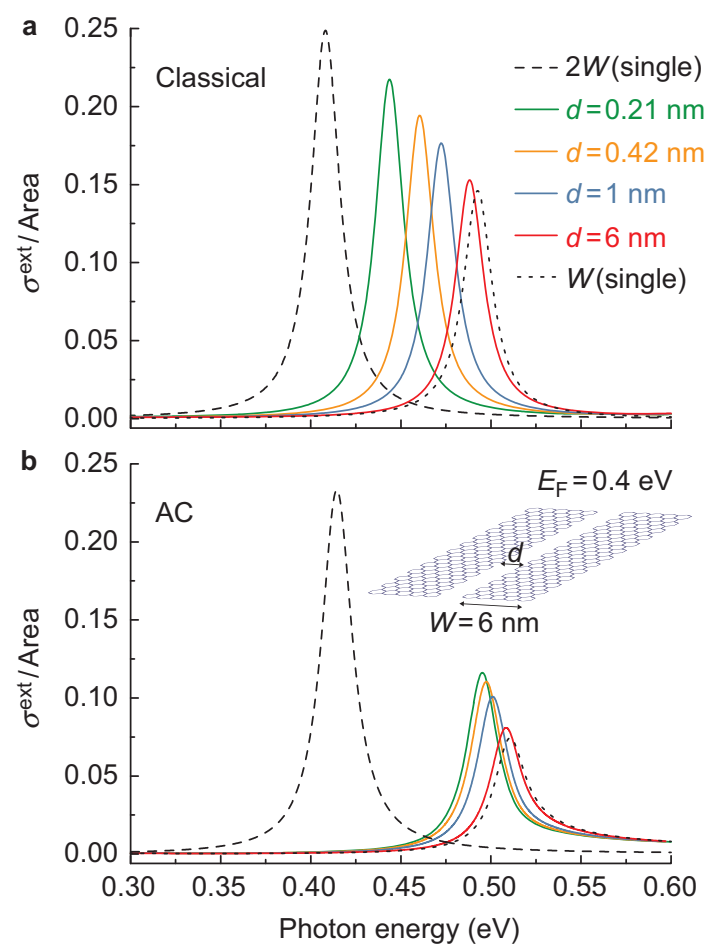

Figure 2 Plasmons in graphene ribbon dimers. Normal-incidence classical (a) and quantum-mechanical TB-RPA (AC edges) (b) calculations of the extinction cross-sections of ribbon dimers (width $W=6 \mathrm{~nm}$, Fermi energy $E_{\mathrm{F}}=0.4 \mathrm{eV}$ and intrinsic damping of $0.02 \mathrm{eV}$ ) for different carbon-to-carbon edge separations $d$ (see also Figure $3 \mathrm{~b}$ for the relation of this parameter to the edge-to-edge distance in the classical model). Spectra for single ribbons of widths $W$ and $2 W$ are shown as dotted and dashed curves, respectively. The light polarization is across the ribbons. AC, armchair; RPA, random-phase approximation; TB, tight-binding.

constant. This relatively small level of absorption can be enhanced through patterning and doping. ${ }^{30}$ We explore this possibility in arrays of narrow ribbons such as those of Figures 4 and 5, which show the evolution of the plasmon energies and their associated absorption. Here, we compare $\mathrm{AC}$ and $\mathrm{ZZ}$ ribbons in order to assess the strength of nonlocal effects.

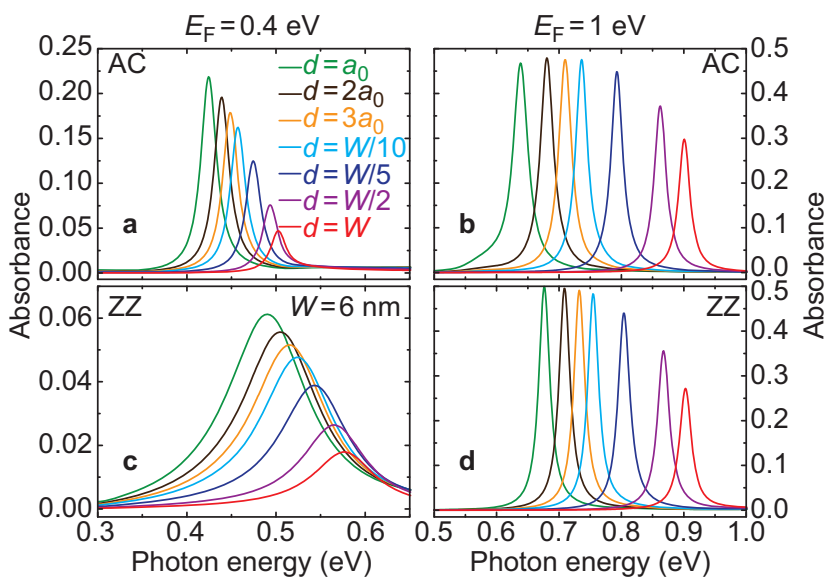

Figure 4 Absorbance of graphene ribbon arrays. We represent the absorbance of $\mathrm{AC}(\mathbf{a}, \mathbf{b})$ and $\mathrm{ZZ}(\mathbf{c}, \mathbf{d})$ ribbon arrays for two different Fermi energies (see upper labels) and various carbon-to-carbon edge separations $d$ (see legend, where we use the $\mathrm{C}-\mathrm{C}$ bond distance $\mathrm{a}_{0}$ ), as calculated from the TB-RPA model. The ribbon width is $W=6 \mathrm{~nm}$ and the intrinsic plasmon damping is $0.02 \mathrm{eV}$ in all cases. AC, armchair; RPA, random-phase approximation; TB, tight-binding; ZZ, zigzag.

The resulting absorption spectra (Figure 4) exhibit again a high sensitivity to $\mathrm{ZZ}$ edges when the plasmon energies are above the Fermi level, leading to a significant reduction of absorption and increasing plasmon broadening (cf. Figure 4a and 4c). At higher doping (Figure $4 \mathrm{~b}$ and $4 \mathrm{~d}$ ), plasmon broadening is limited to the intrinsic damping $(0.02 \mathrm{eV})$ in both $\mathrm{AC}$ and $\mathrm{ZZ}$ ribbon arrays. Interestingly, the inter-ribbon interaction is smaller with $\mathrm{ZZ}$ edges, as revealed by the larger redshift observed in AC ribbons at close separations $d$. In all cases, these shifts are larger than in dimers (cf. for example Figures $2 b$ and $4 \mathrm{a})$. Remarkably, the maximum possible absorption of $50 \%$ is reached with $\mathrm{ZZ}$ edges in Figure $4 \mathrm{~d}$ at short separations $d$ of the order of the $\mathrm{C}-\mathrm{C}$ bond distance, or equivalently, in a structure obtained by removing atomic bonds along an array of parallel rows spaced by a period of $6 \mathrm{~nm}$. Full absorption can further be achieved by placing the structure approximately a quarter wavelength away from a good mirror (Salisbury screen configuration ${ }^{30,52,53}$ ).

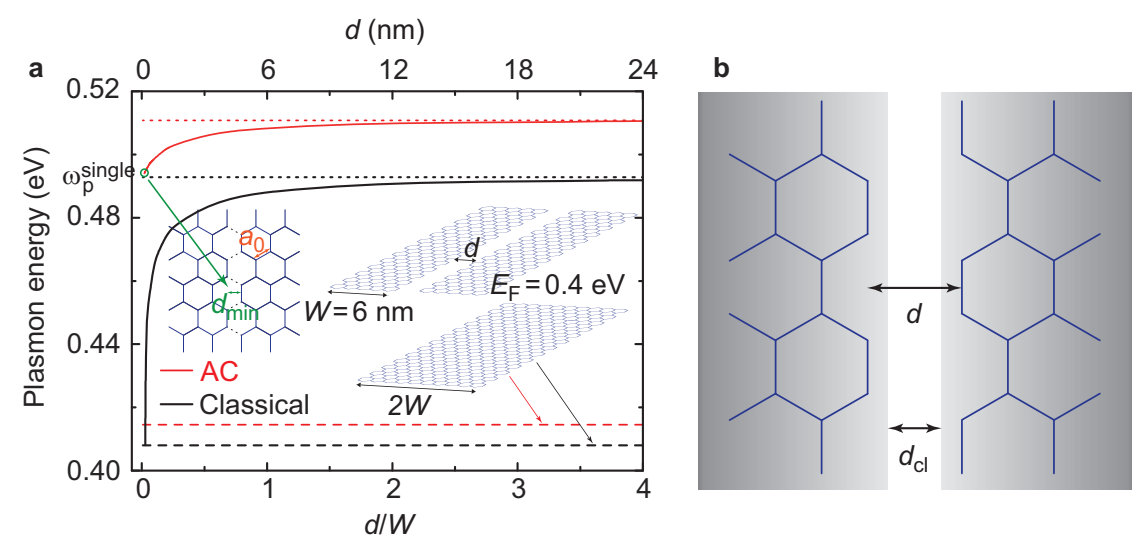

Figure 3 Separation dependence of the transverse plasmon energy in ribbon dimers. (a) We represent the plasmon peak energies extracted from Figure 2 as a function of the carbon-to-carbon edge distance $d$ using classical and quantum-mechanical (for AC ribbons) models. The classical calculations converge well to the double-width-ribbon limit for $d<<W$, in contrast to the quantum-mechanical model, which predicts a substantial energy gap. The left inset shows the nearest separation $d_{\min }=\sqrt{3} a_{0} / 2$ considered for the quantum AC calculations, where $a_{0}=0.1421 \mathrm{~nm}$ is the C-C bond distance. (b) Scheme of the notation used for the separation distance between ribbons: we take $d$ to be the carbon-carbon spacing throughout this paper; classical calculations are performed with a separation between electronic edges given by $d_{\mathrm{Cl}}=d-d_{\min }$. AC, armchair. 

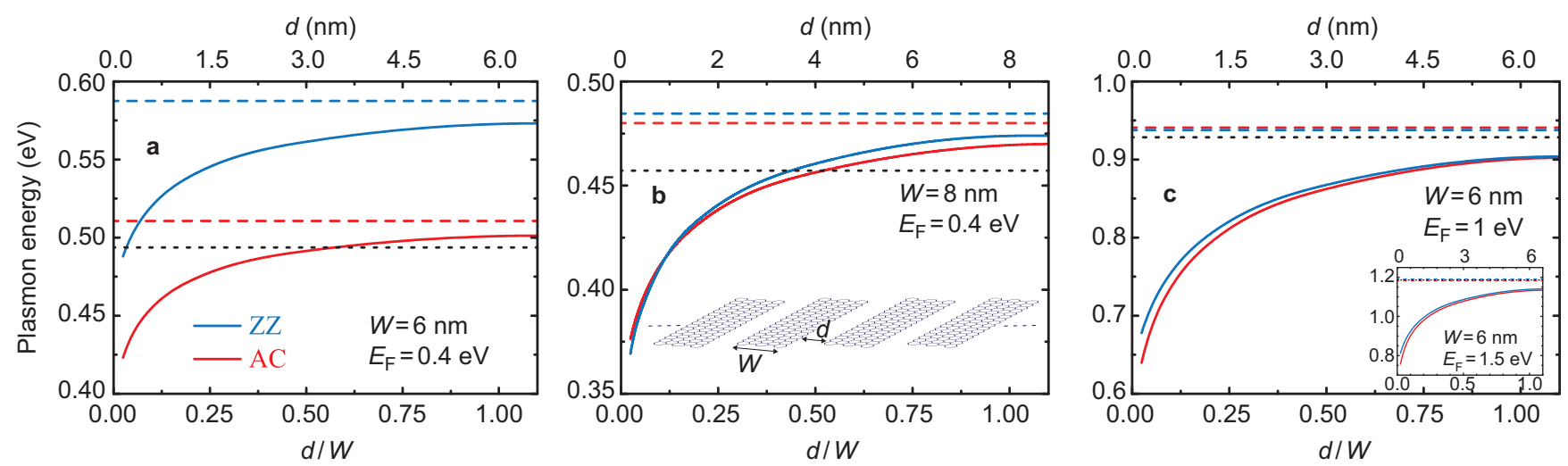

Figure 5 Dependence of the fundamental plasmon mode on inter-ribbon distance in arrays. We show the normal-incidence dipolar plasmon frequency in AC (red curves) and ZZ (blue curves) ribbon arrays as a function of carbon-to-carbon edge separation $d$ for various combinations of ribbon width $W$ and Fermi energy $E_{F}$ (see labels). The dashed lines indicate the single-ribbon limit for each type of edge, whereas the dotted black lines show the classical single-ribbon result. AC, armchair; ZZ, zigzag.

The plasmon energies obtained from TB-RPA in ribbon arrays are summarized in Figure 5, where we confirm that ZZ-edge-induced plasmon quenching does not affect plasmons of energies below the Fermi level. This is clearly illustrated when moving from ribbons of width $W=6 \mathrm{~nm}$ to $W=8 \mathrm{~nm}$ for constant doping $\left(E_{\mathrm{F}}=0.4 \mathrm{eV}\right)$, which causes the plasmon energy to go below $E_{\mathrm{F}}$, giving rise to very similar plasmon energies in both $\mathrm{AC}$ and $\mathrm{ZZ}$ ribbons (Figure 5b). It should be stressed that in the touching limit ( small $d$ ), the graphene becomes an extended sheet that presents nearly zero absorption (at photon energies below $2 E_{\mathrm{F}}$ ), and thus, Figure 5 shows again a discontinuity between this limit and the configurations with ribbons separated by an atomic distance, right after breaking inter-ribbon $\mathrm{C}-\mathrm{C}$ bonds.

\section{CONCLUSIONS}

Nonlocal effects in the plasmons of doped graphene ribbons exhibit the following general properties: (i) plasmons are quenched by $\mathrm{ZZ}$ edges, unless their energies are below the Fermi level, so that they cannot decay through excitation of electronic edge states; (ii) nonlocal quantum effects (generally observed as blueshifts) increase with decreasing ribbon width, although a classical electromagnetic description produces reasonable results for individual ribbons of widths down to a few nanometers; (iii) nonlocal effects are important in interacting ribbons at short separations, leading to substantial blueshifts in the dipole-active plasmons of closely spaced ribbon dimers, in contrast to classical theory, which predicts a smooth convergence towards the double-width ribbon; (iv) remarkably, the removal of a single row of atoms produces a dramatic increase in the plasmon energy, observed both in dimers and in ribbon arrays; (v) our realistic quantum-mechanical calculations yield plasmon energies that are pushed up to the near-infrared regime for ribbon widths of a few nanometers, similar to those that can be synthesized by chemical self-assembly. ${ }^{46}$ These results provide a solid theoretical background for understanding the interaction between graphene nanoribbons, which should be relevant to the design of graphene-plasmon-based electro-optical modulators and switchers.

\section{ACKNOWLEDGEMENTS}

This work has been supported in part by the European Commission (Graphene Flagship CNECT-ICT-604391 and FP7-ICT-2013-613024-GRASP).
1 Novotny L, Hecht B. Principles of Nano-Optics. New York: Cambridge University Press; 2006

2 Righini M, Volpe G, Girard C, Petrov D, Quidant R. Surface plasmon optical tweezers: tunable optical manipulation in the femtonewton range. Phys Rev Lett 2008; 100 186804.

3 Danckwerts M, Novotny L. Optical frequency mixing at coupled gold nanoparticles Phys Rev Lett 2007; 98: 026104.

4 Davoyan AR, Shadrivov IV, Kivshar YS. Nonlinear plasmonic slot waveguide. Opt Express 2008; 16: 21209-21214.

5 Palomba S, Novotny L. Nonlinear excitation of surface plasmon polaritons by fourwave mixing. Phys Rev Lett 2008; 101: 056802.

6 Xu H, Bjerneld EJ, Käll M, Börjesson L. Spectroscopy of single hemoglobin molecules by surface enhanced Raman scattering. Phys Rev Lett 1999; 83: 4357-4360.

7 Rodríguez-Lorenzo L, Alvarez-Puebla RA, Pastoriza-Santos I, Mazzucco S, Stéphan 0 et al. Zeptomol detection through controlled ultrasensitive surface-enhanced Raman scattering. J Am Chem Soc 2009; 131: 4616-4618.

8 Zia R, Schuller JA, Chandran A, Brongersma ML. Plasmonics: the next chip-scale technology. Mater Today 2006; 9: 20-27.

9 Hwang EH, Das Sarma S. Dielectric function, screening, and plasmons in twodimensional graphene. Phys Rev B 2007; 75: 205418.

10 Ju L, Geng B, Horng J, Girit C, Martin M et al. Graphene plasmonics for tunable terahertz metamaterials. Nat Nanotechnol 2011; 6: 630-634.

11 Fei Z, Andreev GO, Bao W, Zhang LM, McLeod AS et al. Infrared nanoscopy of dirac plasmons at the graphene- $\mathrm{SiO}_{2}$ interface. Nano Lett 2011; 11: 4701-4705.

12 Grigorenko AN, Polini M, Novoselov KS. Graphene plasmonics. Nat Photonics 2012; 6: 749-758.

13 Chen J, Badioli M, Alonso-González P, Thongrattanasiri S, Huth F et al. Optical nanoimaging of gate-tunable graphene plasmons. Nature 2012; 487: 77-81.

14 Fei Z, Rodin AS, Andreev GO, Bao W, McLeod AS et al. Gate-tuning of graphene plasmons revealed by infrared nano-imaging. Nature 2012; 487: 82-85.

15 Yan H, Li X, Chandra B, Tulevski G, Wu Y et al. Tunable infrared plasmonic devices using graphene/insulator stacks. Nat Nanotechnol 2012; 7: 330-334.

16 Fang Z, Thongrattanasiri S, Schlather A, Liu Z, Ma L et al. Gated tunability and hybridization of localized plasmons in nanostructured graphene. ACS Nano 2013; 7: 2388-2395.

17 Brar VW, Jang MS, Sherrott M, Lopez JJ, Atwater HA. Highly confined tunable midinfrared plasmonics in graphene nanoresonators. Nano Lett 2013; 13: 2541-2547.

18 Yan H, Low T, Zhu WJ, Wu YQ, Freitag M et al. Damping pathways of mid-infrared plasmons in graphene nanostructures. Nat Photonics 2013; 7: 394-399.

19 Rodin AS, Fei Z, McLeod AS, Wagner M, Castro Neto AH et al. Plasmonic hot spots in triangular tapered graphene microcrystals. arXiv:1309.1909.

20 Mikhailov SA. Non-linear electromagnetic response of graphene. Europhys Lett 2007; 79: 27002

21 Mikhailov SA. Electromagnetic response of electrons in graphene: non-linear effects. Physica E 2008; 40: 2626-2629.

22 Hendry E, Hale PJ, Moger J, Savchenko AK, Mikhailov SA. Coherent nonlinear optical response of graphene. Phys Rev Lett 2010; 105: 097401.

23 Mikhailov SA. Theory of the giant plasmon-enhanced second-harmonic generation in graphene and semiconductor two-dimensional electron systems. Phys Rev B 2011; 84: 045432

24 Wallace PR. The band theory of graphite. Phys Rev 1947; 71: 622-634.

25 Castro Neto AH, Guinea F, Peres NMR, Novoselov KS, Geim AK. The electronic properties of graphene. Rev Mod Phys 2009; 81: 109-162.

26 Efetov DK, Kim P. Controlling electron-phonon interactions in graphene at ultrahigh carrier densities. Phys Rev Lett 2010; 105: 256805.

27 Chen CF, Park CH, Boudouris BW, Horng J, Geng B et al. Controlling inelastic light scattering quantum pathways in graphene. Nature 2011; 471, 617-620. 
28 Koppens FHL, Chang DE, García de Abajo FJ. Graphene plasmonics: a platform for strong light-matter interactions. Nano Lett 2011; 11: 3370-3377.

29 Fang Z, Wang Y, Schlather AE, Liu Z, Ajayan PM et al. Active tunable absorption enhancement with graphene nanodisk arrays. Nano Lett 2014; 14: 299-304.

30 Thongrattanasiri S, Koppens FHL, García de Abajo FJ. Complete optical absorption in periodically patterned graphene. Phys Rev Lett 2012; 108: 047401.

31 Wunsch B, Stauber T, Sols F, Guinea F. Dynamical polarization of graphene at finite doping. New J Phys 2006; 8: 318.

32 Jablan M, Buljan H, Soljacić M. Plasmonics in graphene at infrared frequencies. Phys $\operatorname{Rev} B$ 2009; 80: 245435.

33 Vakil A, Engheta N. Transformation optics using graphene. Science 2011; 332 1291-1294.

34 Nikitin AY, Guinea F, García-Vidal FJ, Martín-Moreno L. Edge and waveguide terahertz surface plasmon modes in graphene microribbons. Phys Rev B2011; 84: 161407(R).

35 García de Abajo FJ. Graphene plasmonics: challenges and opportunities. ACS Photonics 2014; 1: 135-152.

36 Manjavacas A, Thongrattanasiri S, García de Abajo FJ. Plasmons driven by single electrons in graphene nanoislands. Nanophotonics 2013; 2: 139-151.

37 Brey L, Fertig HA. Elementary electronic excitations in graphene nanoribbons. Phys Rev B 2007; 75: 125434.

38 Thongrattanasiri S, Manjavacas A, García de Abajo FJ. Quantum finite-size effects in graphene plasmons. ACS Nano 2012; 6: 1766-1775.

39 García de Abajo FJ. Nonlocal effects in the plasmons of strongly interacting nanoparticles, dimers, and waveguides. J Phys Chem C 2008; 112: 17983-17987.

40 Savage KJ, Hawkeye MM, Esteban R, Borisov AG, Aizpurua J et al. Revealing the quantum regime in tunnelling plasmonics. Nature 2012; 491: 574-577.

41 Christensen J, Manjavacas A, Thongrattanasiri S, Koppens FHL, García de Abajo FJ. Graphene plasmon waveguiding and hybridization in individual and paired nanoribbons. ACS Nano 2012; 6: 431-440.

42 Nikitin A, Guinea F, García-Vidal FJ, Martín-Moreno L. Surface plasmon enhanced absorption and suppressed transmission in periodic arrays of graphene ribbons. Phys $\operatorname{Rev} B$ 2012; 85: 081405 .
43 Strait JH, Nene P, Chan WM, Manolatou C, Tiwari S et al. Confined plasmons in graphene microstructures: experiments and theory. Phys Rev B 2013; 87: 241410(R)

44 Wu J, Pisula W, Müllen K. Graphenes as potential material for electronics. Chem Rev 2007; 107: 718-747.

45 Li X, Wang X, Zhang L, Lee S, Dai H. Chemically derived, ultrasmooth graphene nanoribbon semiconductors. Science 2008; 319: 1229-1232.

46 Cai J, Ruffieux P, Jaafar R, Bieri M, Braun T et al. Atomically precise bottom-up fabrication of graphene nanoribbons. Nature 2010; 466: 470-473.

47 Li B, Tahara K, Adisoejoso J, Vanderlinden W, Mali KS et al. Self-assembled air-stable supramolecular porous networks on graphene. ACS Nano 2013; 7: 10764-10772.

48 Pines D, Nozières P. The Theory of Quantum Liquids. New York: W. A. Benjamin, Inc. 1966.

49 García de Abajo FJ, Howie A. Retarded field calculation of electron energy loss in inhomogeneous dielectrics. Phys Rev B 2002; 65: 115418.

50 Nair RR, Blake P, Grigorenko AN, Novoselov KS, Booth TJ et al. Fine structure constant defines visual transparency of graphene. Science 2008; 320: 1308.

51 Mak KF, Sfeir MY, Wu Y, Lui CH, Misewich JA et al. Measurement of the optical conductivity of graphene. Phys Rev Lett 2008; 101: 196405.

52 Fante RL, McCormack MT. Reflection properties of the salisbury screen. IEEE Trans Antennas Propag 1988; 36: 1443-1454.

53 Engheta N. Thin absorbing screens using metamaterial surfaces. Antennas Propag Soc Int Symp, IEEE 2002; 2: 392-395.

This work is licensed under a Creative Commons Attribution-

NonCommercial-ShareAlike 3.0 Unported License. The images or other third party material in this article are included in the article's Creative Commons license, unless indicated otherwise in the credit line; if the material is not included under the Creative Commons license, users will need to obtain permission from the license holder to reproduce the material. To view a copy of this license, visit http://creativecommons.org/licenses/by-nc-sa/3.0/ 